Deutscher Nachhaltigkeitskodex

\title{
Vom Vertrauen in den Markt geblendet
}

\begin{abstract}
Der Rat für Nachhaltige Entwicklung beschloss im Oktober 2011 nach zweijährigem Dialogprozess den Deutschen Nachhaltigkeitskodex (DNK). Er gab damit die Empfehlung an die Bundesregierung, sich bei den Unternehmen für eine freiwillige Anwendung des DNK einzusetzen. Von Werner Wild
\end{abstract}

M it dem Deutschen Nachhaltigkeitskodex (DNK) unternimmt der Rat für Nachhaltige Entwicklung (RNE) den Versuch, einen „Standard für Transparenz über Nachhaltigkeitsmanagement von Unternehmen“ zu generieren.

Nach einem zweijährigen Dialog-Prozess mit diversen Stakeholdern liegt seit Oktober 2011 der Nachhaltigkeitskodex vor. Unternehmen, die sich in ihrer Berichterstattung an diesem Kodex orientieren, sollen Informationen zu den Themenbereichen Unternehmensführung, Umwelt und Gesellschaft veröffentlichen. Konkretisiert werden diese Themenfelder durch Daten zu rund 25 Leistungsindikatoren. Nach Vorlage des Entwurfs ging es zuletzt in erster Linie um den Geltungsbereich und die Möglichkeiten zur Implementierung des Deutschen Nachhaltigkeitskodex.

\section{Vorschläge zur Implemen- tierung des DNK}

Die Vorschläge zur Einbeziehung des Nachhaltigkeitskodex in die Unternehmensdarstellung reichten von der verpflichtenden Aufnahme in den Lagebericht über die Prüfung durch Wirtschaftsprüfer bis zur freiwilligen Leistung der Unternehmen in Form einer Selbstauskunft sowie Aufbau einer Transparenzplattform im Internet.

Vertreter von Unternehmen und Wirtschaftsverbänden beteiligten sich intensiv am Dialogprozess. Und es verwundert nicht, dass gerade diese Interessengrup- pen verbindlichen Regelungen kritisch bis ablehnend gegenüber standen. Unternehmen und ihre Verbände plädierten stets für Freiwilligkeit und Selbstauskunft. In diesem Sinne entschied letztlich auch der Rat für Nachhaltige Entwicklung. Die Empfehlung des Rats lautet, die Anwendung des Nachhaltigkeitskodex den Unternehmen zu überlassen. Damit ist erst einmal die im Dialogprozess von verschiedenen Organisationen geforderte rechtliche Verbindlichkeit vom Tisch. Und der Nachhaltigkeitsrat als Beratungsgremium hat sich der Position der Bundesregierung angeschlossen, die die freiwillige Berichterstattung immer wieder betont, wie zuletzt im Fortschrittsbericht 2012 zur nationalen Nachhaltigkeitsstrategie.

\section{Freiwilligkeit setzt auf Marktvertrauen}

Mit der Entscheidung für Freiwilligkeit läuft die anfangs geäußerte Absicht des Nachhhaltigkeitsrats weitgehend ins Leere, mit dem DNK eine verbindliche Regelung für die Unternehmen zu schaffen. Das Vertrauen in den Markt, verbunden mit der Erwartung, dass die Unternehmen aus eigenem Interesse heraus über ihre Nachhaltigkeitsleistung berichten werden, hat den Charakter eines Appells. Es stellt sich die Frage, worin der Mehrwert dieser freiwilligen Information gegenüber den bisherigen Verfahren liegen soll. Verbindlichkeit kann sich nicht in der freiwilligen Darstellung der unter- nehmerischen Verantwortung erschöpfen. Es muss doch für den weiteren Prozess einer nachhaltigen Entwicklung auf Seiten der Unternehmen darum gehen, verpflichtend relevante Informationen zu kommunizieren. Es stehen ja keine Vorschriften für die Unternehmenspolitik und konkrete Handlungsanleitungen für die Unternehmenspraxis zur Debatte, sondern eine Berichterstattung über die Wahrnehmung von Verantwortung für eine nachhaltige Entwicklung.

Die Europäische Kommission verfolgt zurzeit die Absicht, die Berichterstattung über Nachhaltigkeitsleistung von Unternehmen verbindlicher zu gestalten. Die Entscheidung des Rats für Nachhaltige Entwicklung, auf freiwillige Selbstauskunft zu vertrauen, ist für die Perspektive eines europäischen Nachhaltigkeitskodex kein gutes Signal. Einzig das vom Nachhaltigkeitsrat beabsichtigte Monitoring, damit verbunden die Wirksamkeit des Nachhaltigkeitskodex im Markt zu überprüfen und dieses Instrument bis 2013 zu evaluieren, vermittelt einen kleinen Hoffnungsschimmer.

Es wird sich zeigen, ob der Nachhaltigkeitskodex auf breite Akzeptanz stößt und Unternehmen sich dazu verpflichten, vergleichbare Informationen zu den nichtfinanziellen Leistungsindikatoren $\mathrm{zu}$ veröffentlichen. Schließlich stellt sich die Frage, wie Unternehmen ihre Verantwortung für die Gesellschaft wahrnehmen. Eine glaubwürdige und transparente Rechenschaftslegung gehört zum Inhalt von Verantwortung.

- AUTOR + KONTAKT Dr. Werner Wild ist ehrenamtliches Mitglied im BUND Arbeitskreis Wirtschaft und Finanzen und beteiligte sich am Multistakeholderdialog zum DNK.

Hauptberuflich ist er Professor für Allgemeine Betriebswirtschaftslehre, Umwelt- und Nachhaltigkeitsmanagement an der Georg-SimonOhm-Hochschule Nürnberg.

Georg-Simon-Ohm-Hochschule Nürnberg, Fakultät Betriebswirtschaft, Bahnhofstraße 87 , 90402 Nürnberg. Tel.: +49 911 5880-2833, E-Mail:werner.wild@ohm-hochschule.de 


\section{Lizenzhinweis}

Die Beiträge in ÖkologischesWirtschaften werden unter der Creative-Commons-Lizenz "CC 4.0 Attribution Non-Commercial No Derivatives" veröffentlicht. Im Rahmen dieser Lizenz muss der Autor/Urheber stets genannt werden, das Werk darf nicht bearbeitet, abgewandelt oder in anderer Weise verändert und außerdem nicht kommerziell genutzt werden. Die digitale Version des Artikels bleibt für zwei Jahre Abonnent/innen vorbehalten und ist danach im Open Access verfügbar. 